\title{
A MICROBUBBLE-POWERED BIOPARTICLE ACTUATOR
}

\author{
Rebecca A. Braff, Antimony L. Gerhardt, and Martin A. Schmidt \\ Microsystems Technology Laboratories, Massachusetts Institute of Technology \\ Cambridge, MA 02139 \\ Martha L. Gray \\ Division of Health Sciences and Technology, Harvard-MIT \\ Cambridge, MA 02139 \\ Mehmet Toner \\ Center for Engineering in Medicine, Mass. Gen. Hosp., and Health Sciences and Technology, Harvard-MIT
Boston, MA 02114
}

\begin{abstract}
In this paper, we present the results of a device that uses controllable microbubble actuation to manipulate bioparticles. The goal of this project was to design a device that can capture bioparticles (e.g. cells) in predetermined locations and then selectively release them. We use a thermal bubble actuation method to accomplish this goal. In this paper we describe two specific accomplishments: 1) the use of micromachined nucleation cavities and specialized coatings to precisely localize thermal bubbles and to achieve controllable bubble formation temperatures, and 2) the demonstration of controllable microbubbles in a new device for particle sorting.
\end{abstract}

\section{INTRODUCTION}

Microelectromechanical systems (MEMS) have great potential in the biomedical field [1]. Microscale devices can be used for clinical applications such as drug or blood testing, and also for basic biological research into cells and DNA sequencing. While these devices can take advantage of small sample sizes and high throughput that are not possible on the macroscale, there are still significant obstacles that must be overcome to make MEMS devices feasible for most biomedical applications. One of the most critical issues for biological MEMS is the movement and control of fluids and particles in fluids on the microscale.

There are several methods of microfluidic actuation that are currently in use[2-4]. Examples include thermopneumatic pumping $[5,6]$, electrostatic actuation $[7,8]$, piezoelectric actuation[9], bimetalic structures [10], electromagnetic actuation $[\mathbf{1 1}, \mathbf{1 2}]$, and stimuli-responsive hydrogels [13].

Recently, microfabricated devices have been proposed that employ thermally formed microbubbles as actuators, valves, and pumps [5, 14-19]. Microbubble powered devices have the advantage that they can run using relatively uncomplicated electronics, resulting in simple yet robust MEMS. These devices have no moving mechanical parts, and have relatively simple electronics.

The earliest use of bubble formation to create a jet of fluid was in the inkjet printer industry [20-23]. By using a thin-film heater to form a vapor bubble, thermal inkjet pens fire drops of ink out of chambers due to the volume expansion created by the bubble.

Evans et al. used vapor bubbles as valves and pumps in their micromixer[16] and in their 'bubble spring and channel valve' [24]. However, the group reports that the valve may only be opened once because of difficulties removing the initial bubble from the confinement region. This case illustrates some of the problems with the use of microbubbles; namely that bubbles do not always dissipate when the heat is turned off, and that devices are unable to properly manipulate the bubbles to place them in desired locations. This group later used electrochemical bubbles instead of vapor bubbles in a device [25].
Vapor bubbles have also been used as a means of mechanical actuation. Lin et al. $[\mathbf{1 7}, \mathbf{1 8}]$ used microfabricated polysilicon resistive heaters to boil Fluorinert liquid and form a vapor bubble underneath a microfabricated paddle.

A similar application of bubbles in the literature is in a bubble jet that ejects a solution of DNA onto a glass surface[26]. Using this technique, precise volumes of DNA solution may be applied to a surface in order to form a DNA microarray for biological screening.

These examples illustrate the potential of bubble actuation. The explosive vaporization used in the inkjet printing industry has already been proven as an effective, reliable fluid actuation mechanism. Problems still impede the use of controllable microbubbles for fluidic actuation, but if they can be solved, the resulting technology could be very powerful. There has not previously been an experimental or theoretical framework for design and implementation of bubble systems with robust operation characteristics. For microbubbles to be a useful tool for MEMS devices, it is necessary to be able to form bubbles in predetermined locations while minimizing the power necessary to do so, and to be able to do this in a controllable way. A second important issue is that when the heater used to form a bubble is turned off, the bubble often remains due to dissolved gas that has come out of solution into the bubble. This can severely impede the performance of a microbubble-powered device.

In this paper, we present a resistive heater that can form bubbles in predetermined locations, repeatably, and at a reduced superheat from conventional thin-film heaters. We then demonstrate the operation of this heater in a novel bioparticle manipulation device.

\section{DEVICE OVERVIEW}

To establish proof of principle, our goal is to create a device capable of capturing and releasing bioparticles in a controlled fashion, and more specifically to have the potential of scaling it up into a large-scale array. Figure 1 shows our design of the microbubble-powered bioparticle actuator.

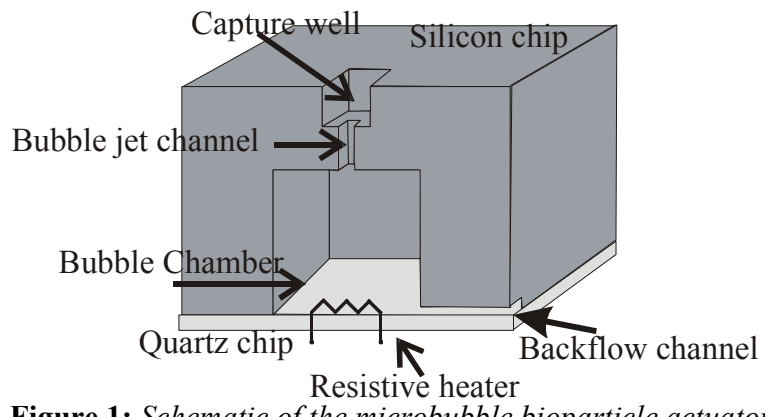

Figure 1: Schematic of the microbubble bioparticle actuator.

Travel support has been generously provided by the Transducers Research

Foundation and by the DARPA MEMS and DARPA Bioflips programs. 
In Figure 2 the operation of the device is shown. When a back flow is applied, a bioparticle may be drawn into a capture well (a). The well can be sized to accommodate only one particle. Then, when a bulk flow is applied over the top of the device, all the uncaptured particles are swept away (b). In order to release the particle, a voltage is applied to the resistive heater in the bubble chamber below and a bubble forms (c). The volume expansion in the bubble chamber pushes out a jet of fluid that ejects the bioparticle from the capture well where it may be entrained in the flow and carried out of the chamber (d). In this paper, we first present the fabrication processes, then discuss the control of the bubble formation process, and finally move on to present the implementation of the microbubble-powered bioparticle actuator.

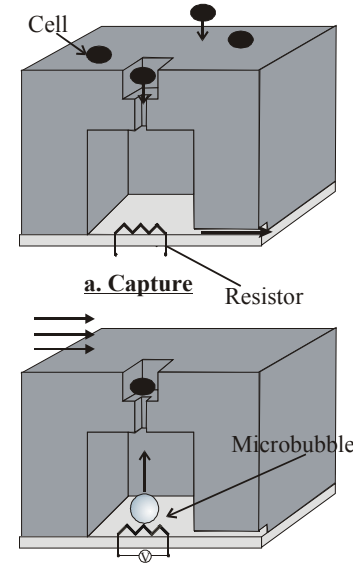

c. Bubble Formation

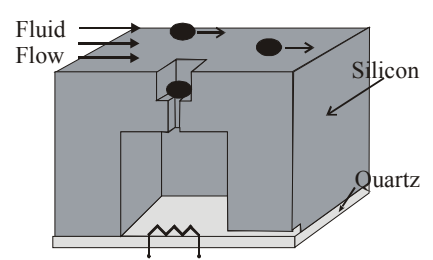

b. Hold

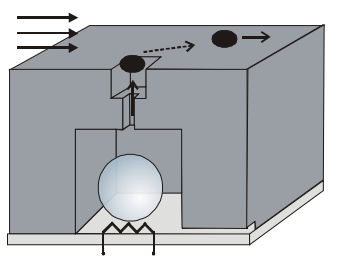

d. Release
Figure 2: Operation of the microbubble bioparticle actuator.

\section{EXPERIMENTAL}

As discussed above, the goals of this work are to first accomplish controllable microbubble formation, and then to demonstrate this bubble actuation in a bioMEMS device.

In order to better control microbubble formation, it is necessary first to understand the bubble nucleation process. When liquid is heated in the presence of a solid surface, heterogeneous nucleation usually occurs. In this regime, bubbles typically nucleate in cavities (surface defects) on the heated surface. The degree of superheat necessary to nucleate a bubble in a cavity is inversely dependent on the cavity radius, as shown in Equation 1.

$$
T_{w}-T_{s a t}=\frac{2 \sigma T_{s a t}}{h_{l v} \rho_{v} r_{c}}
$$

Where $\mathrm{T}_{\mathrm{w}}$ is the surface temperature, $\mathrm{T}_{\mathrm{sat}}$ is the saturation temperature $\left(100^{\circ} \mathrm{C}\right.$ for water $), \sigma$ is the surface tension, $h_{\mathrm{fg}}$ is the latent heat of vaporization, $\rho_{v}$ is the vapor density, and $r_{c}$ is the cavity radius. For example, the surface temperature necessary to nucleate bubbles in water with a surface that has a $1 \mu \mathrm{m}$ cavity radius is about $133^{\circ} \mathrm{C}$. For a $0.1 \mu \mathrm{m}$ cavity radius the temperature to nucleate a bubble is about $432^{\circ} \mathrm{C}$, well above the thermodynamic superheat limit of water, which is about 270 $320^{\circ} \mathrm{C}$. Above this limit, bubbles will spontaneously form and do not need a surface cavity for nucleation. This is called homogeneous bubble nucleation.

Accordingly, for surfaces with cavity sizes well below $1 \mu \mathrm{m}$, it is likely that homogeneous nucleation will occur since the liquid will reach the superheat limit before a bubble nucleates in a cavity. Micromachined surfaces tend to have very smooth surfaces. For instance, for platinum resistors that are only $3-6 \mu \mathrm{m}$ wide, and
$0.1 \mu \mathrm{m}$ thick, it is unlikely that cavities will exist on the surface that are large enough for heterogeneous nucleation to occur.

For this reason, a process was developed whereby cavities approximately $6 \mu \mathrm{m}$ wide and twice as deep could be formed in the middle of platinum thin film heaters on quartz wafers. These cavities could then act as nucleation sites for bubble formation. The process we developed to etch cavities $(5 \mu \mathrm{m}$ square, $10-20 \mu \mathrm{m}$ deep) in quartz wafers and then deposit thin-film platinum resistors over the top is shown in Figure 3. We start with 4 inch quartz wafers and deposit $0.5 \mu \mathrm{m}$ of polysilicon on top of them. We then pattern the cavities using standard photolithography, and etch the pattern into the polysilicon using a plasma etch. Next the quartz is etched, using the polysilicon as a hard mask, with a $\mathrm{CHF}_{3}$ plasma for 75-130 minutes depending on the desired depth. After this the polysilicon mask is stripped in $\mathrm{SF}_{6}$ plasma and the resistors are patterned with photoresist for a lift-off process. A $100 \AA$ adhesion layer of titanium followed by $1000 \AA$ of platinum is deposited by an electron beam tool, then the metal is lifted off in acetone. The final step is to anneal the wafers at $600^{\circ} \mathrm{C}$ in nitrogen for one hour.

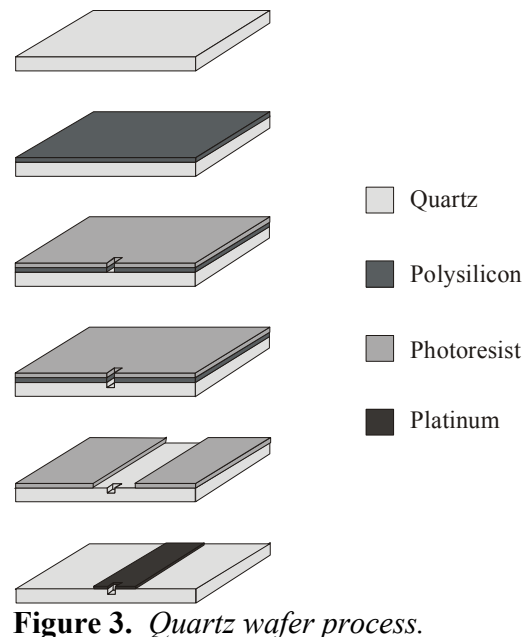

These quartz resistor wafers were tested using a probe station and a semiconductor parameter analyzer to ramp up the voltage while measuring the current in the resistor. The platinum resistors were calibrated to determine the temperature/resistance characteristic so that the temperature of the resistors could be determined from the resulting I-V curves. Resistor wafers were fabricated with and without bubble nucleation cavities and then some of them were coated with $\mathrm{CYTOP}^{\mathrm{TM}}$ [27] or silanized to render the surfaces hydrophobic. Testing was then carried out using a thin layer of deionized water which had been degassed just prior to testing by boiling it for several minutes. A glass cover slip was placed over several drops of this water during testing, in order to prevent evaporation. Voltage was ramped up a half volt at a time, every 1 millisecond until a bubble was formed, and then immediately turned off.

The second phase of testing took place using the full device. The microbubble bioparticle actuator is comprised of two wafers: the quartz resistor wafer described above, and a silicon device wafer, which are bonded together using CYTOP ${ }^{\mathrm{TM}}$. The silicon device wafer fabrication process is shown in Figure 4. The wafer is etched with a nested mask to define the capture well and bubble jet channel on one side, then the heater chamber is etched from the back. Afterwards the wafers are diced into chips and bonded to the quartz resistor chips using CYTOP ${ }^{\mathrm{TM}}$. The method for CYTOP ${ }^{\mathrm{TM}}$ bonding is to prebake a quartz chip on a hotplate at $90^{\circ} \mathrm{C}$ for 30 
minutes, then spin on the $\mathrm{CYTOP}^{\mathrm{TM}}$ at $2000 \mathrm{rpm}$ for 30 seconds, then postbake on a hotplate at $90^{\circ} \mathrm{C}$ for 30 minutes[29]. Immediately afterwards, the silicon chip is bonded to the CYTOPcoated quartz chip using an aluminum jig on top of a hotplate at $160^{\circ} \mathrm{C}$. The chips are aligned and bonded in the jig with a $10 \mathrm{Kg}$ weight on top for 2 hours, then cooled for another hour before removal from the jig.

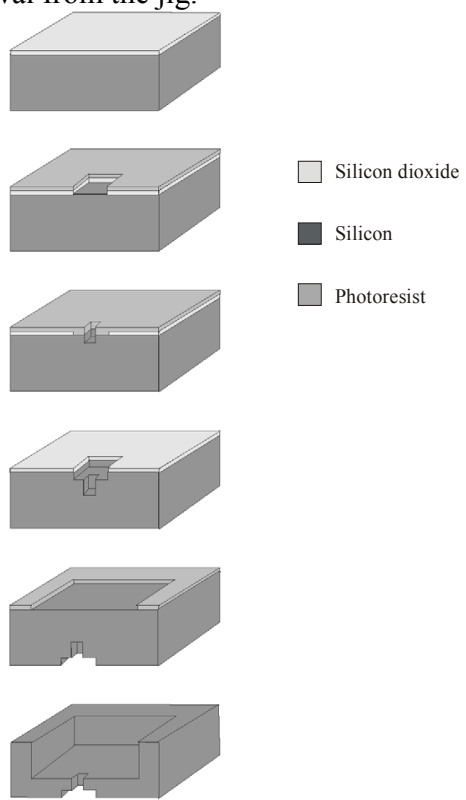

Figure 4. Silicon fabrication process.

A flow chamber was machined to test the devices and is shown in Figure 5. In addition to the inlet and outlet for fluid across the top of the chip, there is an additional port that can be used to fill the heater chambers or alternately, to provide a pressure drop to draw particles into the capture wells via the backflow channel. An aluminum block (a) machined with inlets and an outlet, and a depression in which the chip can sit. A PDMS gasket (b) rests in the depression. The quartz resistor chip (c) bonded to the silicon chip (d) sits on top of the gasket. Another PDMS gasket (e) is placed on top, which is covered by a glass slide (f). The top aluminum cap $(\mathrm{g})$ is screwed down into the aluminum base to create a fluidic seal.

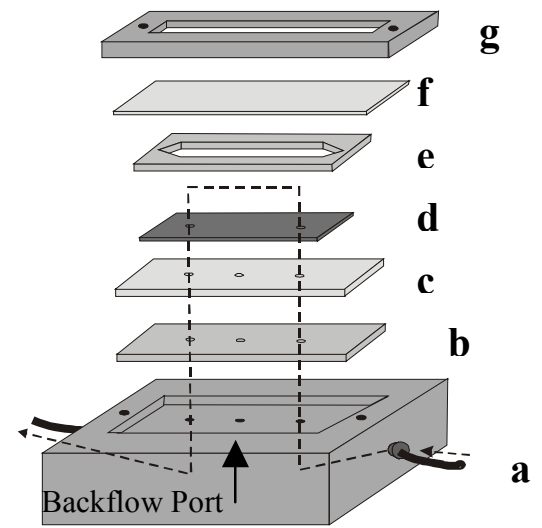

Figure 5. Flow chamber and package.

To test the device, it was first vacuum-filled [28] with $0.05 \%$ Triton X-100 surfactant solution and then placed in the flow chamber. A solution of $10 \mu \mathrm{m}$ diameter polystyrene beads flows over the top of the device, using a syringe pump. Electrical contact is made using probes on a probe station, and bubbles are formed in the bubble chamber using the same method described above for the resistor wafer testing.

\section{RESULTS AND DISCUSSION}

An experiment was run to determine the effect of having cavities in resistive heaters, as well as the effect of the different surface treatments, using resistor wafers prepared six different ways: bare quartz with no cavities, bare quartz with cavities, silanized quartz with no cavities, silanized quartz with cavities, CYTOP-coated quartz with no cavities, and $\mathrm{CYTOP}^{\mathrm{TM}}$-coated quartz with cavities. Testing was carried out as described above. The bubbles formed in cavities on every trial with the two hydrophobic surface preparations (Figure 6), but did not often form in cavities on the bare, untreated quartz. The bubble formation temperature results are shown below in Figure 7. 44 resistors were tested on each wafer to comprise the data shown below.
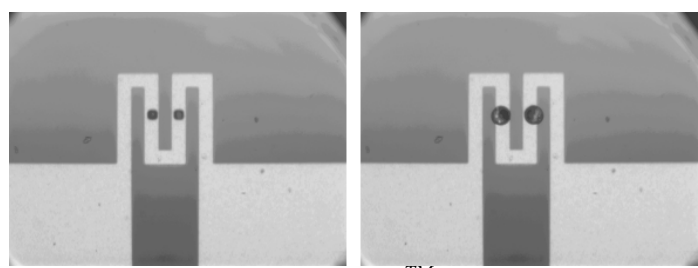

Figure 6: $15 \mu \mathrm{m}$ wide CYTOP ${ }^{T M}$-coated heater with $10 \mu \mathrm{m}$ wide cavities, before and after voltage applied.

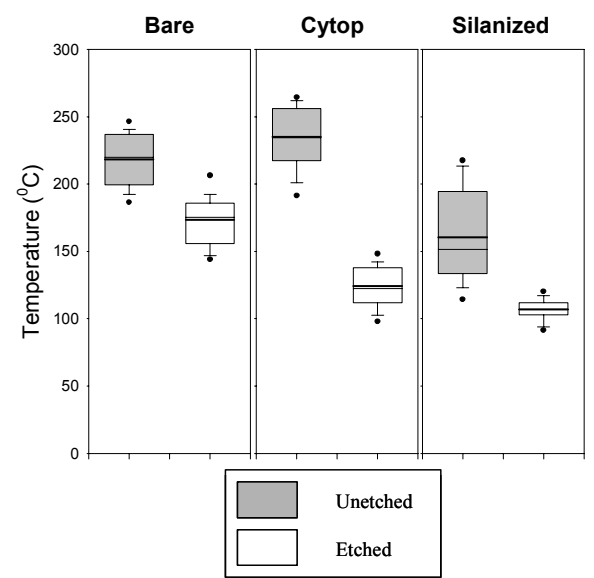

Figure 7: A plot comparing the average bubble formation temperature of bare resistor wafers with and without etched cavities, silanized wafers with and without etched cavities, and $C Y T O P^{T M}$-coated resistor wafers with and without cavities. The boxes represent the $25^{\text {th }}$ through $75^{\text {th }}$ percentiles of data. The lines span the $5^{\text {th }}$ through $25^{\text {th }}$ and $75^{\text {th }}$ through $95^{\text {th }}$ percentiles of the data and the points represent outlying data. Lines in the boxes represent mean and median data.

In all cases, the temperature of bubble formation of a wafer with cavities was lower than the temperature of bubble formation for a wafer prepared the same way with no cavities. As the bubbles formed in holes for the two treated wafers, it makes sense that they formed at lower temperatures. Since the CYTOP ${ }^{\mathrm{TM}}$ layer is $1 \mu \mathrm{m}$ thick, considerably thicker than the effect of silanization, it makes sense that this added thermal resistance would result in a higher bubble formation temperature for the etched wafers coated with CYTOP ${ }^{\mathrm{TM}}$ than for the etched silanized wafers.

The photographs of device operation are shown in Figure 8. In (A) and (B) a small backflow pulls a bead into a well and it is 
then held there against the bulk flow in the chamber (C) $(\mathrm{t}=0)$. In (D) the bead is ejected from the well when a bubble is formed in the chamber below $(\mathrm{t}=0.77 \mathrm{sec})$, and $\mathrm{In}(\mathrm{E})$ and $(\mathrm{F})$ the bead is entrained in the bulk flow and carried away $(\mathrm{t}=0.87, \mathrm{t}=2.66 \mathrm{sec})$.
(A) Capture Wel

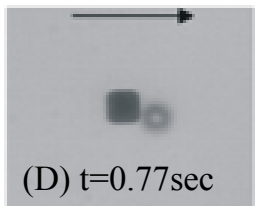

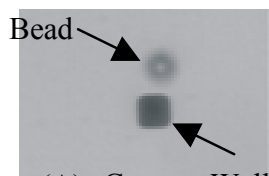
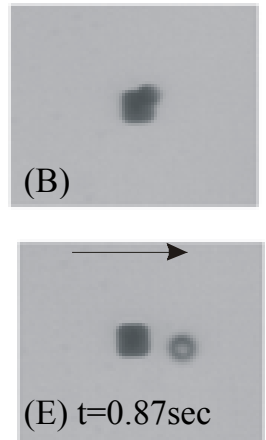
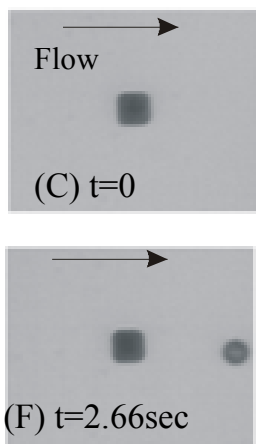

Figure 8: Sequential photos of device operation.

\section{CONCLUSION}

This actuator demonstrates the potential of microbubbles for fluidic actuation. The device has also successfully manipulated yeast cells. The next step will be to create a small array of wells and complete testing with biological cells.

\section{ACKNOWLEDGEMENTS}

This work was carried out with funding from the Alliance for Cellular Signaling project(NIGMS). Fabrication was completed at the MIT Microsystems Technology Laboratories. The authors would also like to thank Professor B. Mikic for many useful discussions about bubble formation. Travel support has been generously provided by the Transducers Research Foundation and by the DARPA MEMS and DARPA bioflips programs.

\section{REFERENCES}

1. Voldman, J., M.L. Gray, and M.A. Schmidt, "Microfabrication in Biology and Medicine." Annual Review of Biomedical Engineering, 1999. 1: p. 401-425.

2. Shoji, S. and M. Esashi, "Microflow Devices and Systems." Journal of Micromechanics and Microengineering, 1994. 4: p. 157-171.

3. Ho, C.-M. and Y.-C. Tai, "Micro-Electro-Mechanical-Systems (MEMS) and Fluid Flows." Annual Review of Fluid Mechanics, 1998. 30: p. 579-612.

4. Gravesen, P., J. Branebjerg, and O.S. Jensen, "Microfluidics- A Review." Journal of Micromechanics and Microengineering, 1993. 3: p. 168-182.

5. Elwenspoek, M., et al., "Towards Integrated Microliquid Handling Systems." Journal of Micromechancs and Microengineering, 1994. 4: p. 227-245.

6. Grosjean, C. and Y.-C. Tai. "A Thermopneumatic Peristaltic Micropump." Transducers '99. 1999.

7. Zengerle, R., et al. "Performance Simulation of Microminiaturized Membrane Pumps." Transducers '93. 1993. 8. Zengerle, R., et al., "A Bidirectional Silicon Micropump." Sensors and Actuators A, 1995. A50: p. 81-86.

9. Koch, M., et al., "A Novel Micromachined Pump Based on Thick-Film Piezoelectric Actuation." Sensors and Actuators A, 1998. 70: p. 98-103.
10. Jerman, H. "Electrically-Activated, Micromachined Diaphragm Valves." IEEE Solid State Sensor and Actuator Workshop. 1990.

11. Miller, R.A., et al. "Electromagnetic MEMS Scanning Mirrors for Holographic Data Storage." Solid State Sensor and Actuator Workshop. 1996.

12. Yanagisawa, K., H. Kuwano, and A. Tago. "An

Electromagnetically Driven Microvalve." Transducers '93. 1993.

13. Liu, R.H., Q. Yu, and D.J. Beebe, "Fabrication and

Characterization of Hydrogel-Based Microvalves." Journal of

Microelectromechanical Systems, 2002. 11(1): p. 45-53.

14. Choi, B.K., et al. "Electrolytic and Thermal Bubble

Generation Using AC Inductive Powering." Transducers '99. 1999.

Sendai, Japan.

15. Donald, D.K., "Thermal Optical Switches for Light." 1998,

Hewlett Packard Company: US Patent 5,732,168.

16. Evans, J., D. Liepmann, and A.P. Pisano. "Planar Laminar Mixer." MEMS '97. 1997.

17. Lin, L., A. Pisano, and A. Lee. "Microbubble Powered Actuator." Transducers '91. 1991.

18. Lin, L. and A. Pisano, "Bubble Forming on a Micro Line Heater," ASME Microechanical Sensors, Actuators, and Systems, D. Cho, et al., Editors. 1991. p. 147-163.

19. Lin, L., "Microscale Thermal Bubble Formation: Thermophysical Phenomena and Applications." Microscale thermophysical Engineering, 1998. 2: p. 71-85.

20. Beatty, C.C. "A Chronology of Thermal Ink-Jet Strucures." Solid-State Sensor and Actuator Workshop. 1996. Hilton Head, S.C.

21. Asai, A., "Application of the Nucleation Theory to the Design of Bubble Jet Printers." Japanese Journal of Applied Physics, 1989. 28: p. 909-915.

22. Allen, R.R., J.D. Meyer, and W.R. Knight, "Thermodynamics and Hydrodynamics of Thermal Ink Jets." Hewlett-Packard Journal, 1985. 36: p. 21-27.

23. Nielsen, N.J., "History of Thinkjet Printhead Development." Hewlett-Packard Journal, 1985. 36: p. 4-10.

24. Evans, J.D. and D. Liepmann. "The Bubble Spring and Channel (BSAC) Valve: An Actuated, Bistable Mechanical Valve for In-Plane Fluid Control." Transducers '99. 1999. Sendai, Japan. 25. Papavasiliou, A.P., D. Liepmann, and A.P. Pisano.

"Electrolysis-Bubble Actuated Gate Valve." Solid-State Sensor and Actuator Workshop. 2000.

26. Okamoto, T., T. Suzuki, and N. Yamamoto, "Microarray Fabrication with Covalent Attachment of DNA Using Bubble Jet Technology." Nature Biotechnology, 2000. 18: p. 438-441. 27. Matsumoto, Y., K. Yoshida, and M. Ishida, "A Novel Deposition Technique for Fluorocarbon Films and its Applications for Bulk- and Surface-Micromachined Devices." Sensors and Actuators A, 1998. 66: p. 308-314.

28. Monahan, J., A.A. Gewirth, and R.G. Nuzzo, "A Method for Filling Complex Polymeric Microfluidic Devices and Arrays." Analytical Chemistry, 2001.73: p.3193-3197.

29. Lu, H., Schmidt, M.A., Jensen, K.F., "Photochemical Reactions and On-Line Detection in Microfabricated Reactors." Lab on a Chip, 2001 1:p.22-28. 